\title{
Specific growth rate estimation in (fed-)batch bioreactors using second-order sliding observers
}

\author{
Hernán De Battista ${ }^{\mathrm{a}, *}$, Jesús Picó ${ }^{\mathrm{b}}$, Fabricio Garelli ${ }^{\mathrm{a}}$, Alejandro Vignoni $^{\mathrm{b}}$ \\ a CONICET, LEICI, Facultad de Ingeniería, Universidad Nacional de La Plata, La Plata, Argentina \\ b Institut d'Automàtica i Informàtica Industrial, Universitat Politècnica de València, Spain
}

\section{A R T I C L E I N F O}

Article history:

Received 30 November 2010

Received in revised form 20 May 2011

Accepted 21 May 2011

Available online 15 June 2011

\section{Keywords:}

Bioreactors

Process control

Nonlinear observers

Sliding mode

Fermentation processes

\begin{abstract}
A B S T R A C T
This paper addresses the estimation of specific growth rate of microorganisms in bioreactors using sliding observers. In particular, a second-order sliding observer based on biomass concentration measurement is proposed. Differing from other proposals that only guarantee bounded errors, the proposed observer provides a smooth estimate that converges in finite time to the time-varying parameter. Stability is proved using a Lyapunov approach. The observer exhibits also robustness to process uncertainties since no model of the reaction is used for its design. In addition, the off-surface coordinate of the sliding observer is useful to determine the convergence time as well as to identify sensor faults and unexpected behaviors. Because of the structure of the output error injection, chattering phenomena of conventional sliding mode algorithms are substantially reduced. The features of the proposed observer are assessed by numerical and experimental data.
\end{abstract}

(c) 2011 Elsevier Ltd. All rights reserved.

\section{Introduction}

Bioprocesses are characterized by complex dynamic behavior, nonlinearities, model uncertainty, unpredictable parameter variations, etc. Furthermore, most representative variables are typically not accessible for on-line measurement. In this context, the development of robust and reliable algorithms to estimate key variables and parameters is of prime interest, both for process control and monitoring [1].

The existing algorithms differ from each other with respect to the measured and estimated variables, the parameters which are assumed to be known, the type of convergence, robustness issues, etc. A summary of several approaches under different scenarios can be found in $[1,2]$. Asymptotic observers for state and parameter estimations appeared for the first time in [3]. Adaptive high-gain observers for the same purposes were presented in [4]. Applications of high-gain observers to bioreactors were treated also in $[5,6]$. More recently, hybrid observers combining asymptotic with exponential observers to estimate states and identify confidence of the kinetic model were developed [7,8]. A proportional-integral observer based on the exact transformation of the nonlinear dynamical system into its multi-model form has been developed in [9]. Sliding mode observers have been proposed also to deal with model uncertainties $[10,11]$. An observer that

\footnotetext{
* Corresponding author. Tel.: +54 2214259306.

E-mail address: deba@ing.unlp.edu.ar (H. De Battista).
}

estimates the substrate consumption rate based on substrate concentration measurement was designed in [10]. In [11], sliding mode techniques were exploited to estimate kinetic rates and concentration variables from biomass measurement.

In this paper we focus on the estimation of reaction rates and, particularly, of specific growth rates. The motivation is that, in many cases, specifications are related with the growth rate of microorganisms, whether the objective is to maximize biomass production or to maintain a metabolic steady state [12]. Besides, growth rate provides valuable information to monitor the development of microorganisms in the broth.

Substrate concentrations are the key variables in the kinetic models. So, by measuring them, good estimates of the specific growth rate can be obtained by using high-performance observers. However, substrates are usually very difficult to measure on-line and with good precision, particularly when they are in low concentrations.

Alternatively, there currently exist reliable biomass sensors (see for example $[13,14])$. That is why much research has been oriented to develop observers based on biomass sensors, although biomass is a much less informative signal from the point of view of kinetics than substrate. In this approach, the kinetic rate is traditionally treated as an unknown parameter. Advances in the field can be traced back to the work of [4], where an adaptive Luenberger-like observer is designed so that it achieves bounded error under the assumption that the specific growth rate has bounded time derivative. These results were extended and improved by further work of the authors and contributions of other colleagues. 
A different approach is suggested in [11], where reaction rates are treated as unknown time-varying signals rather than as unknown parameters. There, a sliding mode observer is designed to estimate the specific growth rate under the same assumptions as in [4] and related papers. The observer includes a discontinuous term in the estimate that allows achieving finite time convergence to the unknown growth rate. Actually, the estimate converges to the real signal up to a very high frequency component.

In this paper we further exploit the potentialities of the previous approach with the aim of obtaining observers with superior convergence features than the already existing ones. It is actually a super-twisting algorithm [15], modified to deal with the nonlinearities of the process. The information about the process required by the observer is the same as in [4] and related papers, thereby similar robustness features are expected. The present approach has also interesting applications in fault detection and monitoring.

The work is organized as follows. The next section presents some general assumptions and preliminaries. In Section 3, the proposed second-order sliding mode observer is developed and its stability is proved using Lyapunov theory and semi-definite programming tools. Section 4 shows the observer performance using numerical analysis whereas experimental results are presented in Section 5. Finally, the main conclusions of the work and future research lines are given.

\section{Problem formulation and background material}

Consider a biomass growth, whose dynamics accept the following description in state-space [16,17]:

$\mathcal{P}:\left\{\begin{array}{l}\dot{x}=(\mu-D(x, t)) x \\ \dot{\mu}=\rho(x, \mu, t) x\end{array}\right.$

where the state variables are the biomass concentration $x$ and the specific growth rate $\mu$. The dilution rate $D(x, t)$ is a function of time and, possibly, of $x$. The specific growth rate $\mu$ is an unknown nonlinear function of biochemical and environmental variables. In the second line of (1), a biomass-proportional representation for the $\mu$ dynamics has been used. This is a sensible choice, particularly for batch processes as well as for fed-batch processes with exponential growth (in which feeding laws of the form $D(x, t)=\lambda(t) x$, are used). An explicit expression for $\rho(\cdot)$, as function of process parameters, can be derived for some simple - and most commonly found in literature - kinetic models such as Monod and Haldane. However, our purpose is to design robust observers that do not rely on the knowledge of the kinetic structure and process parameters. Therefore, the function $\rho(x, \mu, t)$ is supposed to be unknown.

\subsection{Main assumptions}

The observer to be presented in the following section is designed under the following main assumptions:

Assumption 1. Biomass concentration is measured.

Assumption 2. Uncertainty $\rho$ is uniformly bounded by $|\rho(\cdot)|<\bar{\rho}$

Assumption 3. The dilution rate $D$ is known and uniformly bounded.

Additionally, to show observer convergence, we state the following assumptions which are quite obvious and do not restrict the validity of the proposed observer:

Assumption 4. $D$ and $\rho$ are Lebesgue-measurable functions.

Assumption 5. Biomass concentration is strictly positive and bounded, that is, for any initial condition $x(0)>0$ there exist $\underline{x}>0$ and $\bar{x}<\infty$ such that $\underline{x}<x(t)<\bar{x} \forall t>0$.

\subsection{Preliminaries}

High-gain observers are based on the works of Bastin and Dochain [4]. They have the form

$\mathcal{O}_{B \& D}:\left\{\begin{array}{l}\dot{\hat{x}}=(\hat{\mu}-D(x, t)+2 \zeta \omega(x-\hat{x})) x \\ \dot{\hat{\mu}}=\omega^{2}(x-\hat{x}) x\end{array}\right.$

This is a Luenberger observer for the measured signal $x$ with an integral state that adapts the estimation of the unknown parameter $\mu$. That is, the error in the estimation of a measured variable is used in turn to estimate the unknown parameter. The adaptive observer effectively behaves as a low-pass second-order filter of the unknown growth rate $\mu$. Several tunings, variations and extensions of this observer have been proposed in the literature. In any case, perfect tracking of a time-varying $\mu(t)$ cannot be achieved and only steady state errors in $\hat{\mu}$ can be eliminated. This sort of observer is said to be non-exact in the sense that the real signal cannot be recovered even in the absence of noise. In feedback control loops, these observer dynamics add to the controller dynamics, so that the separation principle does not apply. In the last sections we will use this traditional observer to make a comparative analysis with the proposed sliding one.

On other side, the first-order sliding mode observer for (1) presented in [11] is of the form ${ }^{1}$ :

$$
\mathcal{O}_{1 S M}:\left\{\begin{array}{l}
\dot{\hat{x}}=\left(z-D(x, t)+\omega(1+a(x))(x-\hat{x})+\frac{M}{\omega} \operatorname{sign}(x-\hat{x})\right) x \\
\dot{z}=\left(\omega^{2} a(x)(x-\hat{x})+M \operatorname{sign}(x-\hat{x})\right) x \\
\hat{\mu}=z+\frac{M}{\omega} \operatorname{sign}(x-\hat{x})
\end{array}\right.
$$

with $\omega>0, M \geq \bar{\rho}$ and $a(x) \geq 0 \forall x$.

Note that it has the same form as the B\&D observer, but discontinuous terms are added to the observer dynamics and output. Thus, the estimated biomass perfectly tracks the measured one after a finite converging time, whereas the resulting specific growth rate estimate is discontinuous. Further, this estimate coincides with the real growth rate except for a very high (ideally infinite) frequency discontinuous error. Two options have been explored in [11] to recover the continuous signal from the discontinuous estimate. The first, and most obvious one, consists in passing the observer output through a low-pass filter of arbitrary order and cut-off frequency. In the second one, the discontinuous $\operatorname{sign}(\cdot)$ function is replaced by a continuous function with high gain at the origin. In both cases, the continuous estimate no longer converges in finite time to the real time-varying growth rate but just to a ball centered around. Hence, this observer is not exact either. It is however more flexible and it has been shown to be less noisy in many circumstances than observer (2)[11].

\section{Second-order sliding mode observer}

The new observer differs from (3) in the structure of the discontinuous output error injection. It falls within the category of second-order sliding mode observers since the switching argument must be differentiated twice for discontinuity to appear. Further, the proposed observer is a variation of the super-twisting sliding algorithm and inherits some of its attractive features such as finite-time convergence $[18,19]$ and robustness against noise and discretization [15]. Those readers unfamiliar with high-order sliding modes are referred also to the comprehensive works [20,21].

\footnotetext{
1 [11] proposes also an observer designed to estimate bounded growth rates with unbounded derivatives.
} 
Consider the biomass dynamic system (1), where $\rho$ and $D$ are input signals satisfying Assumptions 3 and 4. Therefore, a well-defined solution exists for any initial condition. Further, any solution to (1) satisfies also the differential inclusion ${ }^{2}$

$\mathcal{P}_{U}\left\{\begin{array}{l}\dot{x}=(\mu-D(x, t)) x \\ \dot{\mu} \in U \bar{\rho} x\end{array}\right.$

where $U$ is the set $U=[-1,+1]$. This differential inclusion represents the family of solutions for any unknown specific growth rate satisfying Assumption 2.

Theorem 1. Let $(x(t), \mu(t))$ be a solution of the differential inclusion (4), with $x(t)$ satisfying Assumption 5. Then, the observer

$\mathcal{O}_{2 S M}:\left\{\begin{array}{l}\dot{\hat{x}}=\left(\hat{\mu}-D(x, t)+2 \beta(\bar{\rho}|(x-\hat{x})|)^{1 / 2} \operatorname{sign}(x-\hat{x})\right) x \\ \dot{\hat{\mu}}=(\alpha \bar{\rho} \operatorname{sign}(x-\hat{x})) x\end{array}\right.$

converges in finite-time to $(x(t), \mu(t))$ for suitable gains $\alpha$ and $\beta$.

Remark 1. Notice that the proposed observer can be used to estimate an unknown signal $r(t)$ in any dynamical system of the form

$\dot{p}=r(t) \cdot p+f(p, t)$

provided $p$ is measured and analogous assumptions to the ones made here are fulfilled.

Note. Convergence is understood here in the sense that the estimation error vanishes for any solution to (4). Note that weaker concepts of convergence are also used in the literature, meaning that the estimation error reaches a neighborhood of the origin for any solution to (4), or that it exponentially or asymptotically approaches the origin for solutions satisfying $\dot{\mu} \rightarrow 0$. Finite-time convergence means that there exists $T<\infty$ such that $(\hat{x}(t), \hat{\mu}(t)) \equiv$ $(x(t), \mu(t)) \forall t>T$.

Convergence of (5) is proved here using a Lyapunov approach together with semi-definite programming tools. To this end, consider the following proposition.

Proposition 1. Let

$\dot{z}=A(t) z, \quad A(t) \in \mathcal{A} \forall t$

be a polytopic linear differential inclusion with

$\mathcal{A}=\operatorname{conv}\left(A_{1}, A_{2}\right)$
$A_{1}=\left[\begin{array}{cc}-\beta & 1 / 2 \\ -(\alpha-1) & 0\end{array}\right]$
$A_{2}=\left[\begin{array}{cc}-\beta & 1 / 2 \\ -(\alpha+1) & 0\end{array}\right]$

Then, for every $\alpha>1$ there exist suitable values of $\beta$ such that (7) is quadratically stable for all $A(t) \in \mathcal{A}$.

Note. The polytopic linear differential inclusion is said quadratically stable if there exists $V(z)=z^{T} P z, P \succ 0$ that decreases along every nonzero trajectory of (7).

Since $\dot{V}(z)=z^{T}\left(A(t)^{T} P+P A(t)\right) z$, a necessary and sufficient condition for quadratic stability is

$$
\begin{gathered}
P \succ 0 \\
A^{T}(t) P+P A(t)+v I \prec 0 \quad \forall A(t) \in \mathcal{A}
\end{gathered}
$$

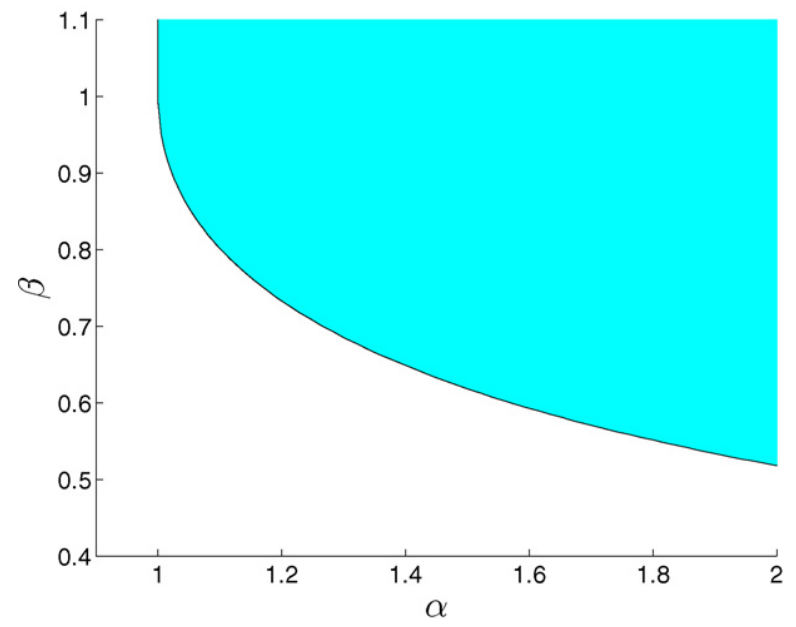

Fig. 1. Set of pairs $(\alpha, \beta)$ for which Proposition 1 holds.

This is equivalent to determine the existence of a common Lyapunov matrix $P$ for all the vertices of the polytope $\mathcal{A}$, i.e. that verifies the following constraints

$\mathcal{F}=\left\{\begin{array}{l}P \succ 0 ; \\ Q_{1} \triangleq-\left(A_{1}^{T} P+P A_{1}\right) \succ 0 ; \\ Q_{2} \triangleq-\left(A_{2}^{T} P+P A_{2}\right) \succ 0\end{array}\right\}$

Now rewriting $A_{1}$ and $A_{2}$ in a convenient way,

$A_{1}=\beta A_{0}+A_{1}^{*}$

$A_{2}=\beta A_{0}+A_{2}^{*}$

where

$A_{0}=\left[\begin{array}{cc}-1 & 0 \\ 0 & 0\end{array}\right]$

$A_{1}^{*}=\left[\begin{array}{cc}0 & 1 / 2 \\ -(\alpha-1) & 0\end{array}\right]$

$A_{2}^{*}=\left[\begin{array}{cc}0 & 1 / 2 \\ -(\alpha+1) & 0\end{array}\right]$

The existence of a common Lyapunov $P$ for any $\alpha$ can be determined by checking the feasibility of the following generalized eigenvalue problem (GEVP) in $P$ and $\beta$ [22]:

$\min \beta$

s.t. $\quad P \succ 0, \quad \beta>0, \quad \mathcal{F}^{*}$

where

$\mathcal{F}^{*}=\left\{\begin{array}{l}P \succ 0 ; \\ \left(A_{1}^{* T} P+P A_{1}^{*}\right)+\beta\left(A_{0}^{T} P+P A_{0}\right) \prec 0 ; \\ \left(A_{2}^{* T} P+P A_{2}^{*}\right)+\beta\left(A_{0}^{T} P+P A_{0}\right) \prec 0 ;\end{array}\right\}$

A GEVP is a quasi-convex problem. In this case, it can be solved using a bisection algorithm on $\beta$ and determining the feasibility of the remaining linear matrix inequality (LMI). We made a grid covering the desired values of $\alpha$ and solved the LMIs with YALMIP [23]. Fig. 1 shows the set of values of $\alpha$ and $\beta$ for which the LMI problem is feasible. For all points within this set of parameters, (7) is quadratically stable. ${ }^{3}$

\footnotetext{
${ }^{3}$ Searching for a common $P$ for all vertices in a polytope may be difficult in some
} situations but not in the current case. 
Proof 1 (of Theorem 1). From the process (4) and observer (5), the error dynamics is

$\left\{\begin{array}{l}\dot{\tilde{x}}=\left(\tilde{\mu}-2 \beta(\bar{\rho}|\tilde{x}|)^{1 / 2} \operatorname{sign}(\tilde{x})\right) x(t) \\ \dot{\tilde{\mu}} \in(U-\alpha \operatorname{sign}(\tilde{x})) \bar{\rho} x(t)\end{array}\right.$

where $\tilde{x} \triangleq(x-\hat{x})$ and $\tilde{\mu} \triangleq(\mu-\hat{\mu})$. Note that it depends on the process state $x$, i.e. it is not an autonomous dynamics. Apply now to (15) the following global homeomorphism [19]

$\xi=\left[\begin{array}{c}(|\bar{\rho} \tilde{x}|)^{1 / 2} \operatorname{sign} \tilde{x} \\ \tilde{\mu}\end{array}\right]$

Taking into account that $\operatorname{sign}\left(\xi_{1}\right)=\operatorname{sign}(\tilde{x})$ and that $\dot{\xi}_{1}=$ $\left(\bar{\rho} / 2\left|\xi_{1}\right|\right) \dot{\tilde{x}}$, this coordinate transformation yields

$\dot{\xi} \in \frac{\bar{\rho} x(t)}{\left|\xi_{1}\right|} \mathcal{A} \xi$

with $\mathcal{A}$ defined in (8). Consider now the energy function $V(\xi)=\xi^{T} P \xi$, where $P \succ 0$ satisfies (9). Then,

$\dot{V}(\xi, t)=\frac{\bar{\rho} x(t)}{\left|\xi_{1}\right|} \xi^{T}\left(A(t)^{T} P+P A(t)\right) \xi$

Using (9) and recalling Assumption 5,

$\dot{V}(\xi, t) \leq-\frac{\bar{\rho} \underline{x} v}{\left|\xi_{1}\right|}\|\xi\|^{2}<0 \forall \xi \neq 0$.

where $v>0$ is the minimum among all eigenvalues of $Q_{1}$ and $Q_{2}$. That is, $V(\xi)$ is a Lyapunov function decreasing along all nonzero solutions of (17). Note that (16) is continuously differentiable everywhere except on the line $\tilde{x}=0$. Anyway, this line is not an invariant set except the origin. Thus, (19) also proves stability of the original observer error dynamics (15).

We will prove now that $V$ vanishes in finite time. Let $L>l>0$ be the maximum and minimum eigenvalues of $P$. Then, $l\|\xi\|^{2} \leq V(\xi$, $t) \leq L\|\xi\|^{2} \forall t$. It then follows, using $\left|\xi_{1}\right| \leq\|\xi\|$, that

$\dot{V} \leq-\frac{\bar{\rho} \underline{x} \nu \sqrt{l}}{L} V^{1 / 2}$

The comparison lemma establishes that any solution $\xi(t)$ to the differential inclusion (17) satisfies

$\|\xi(t)\| \leq \sqrt{\lambda}\|\xi(0)\|-\frac{\bar{\rho} \underline{x} v}{2 L} t$

where $\lambda=\sqrt{L / l}$. This means that the trajectory of the observer error reaches the origin in finite time:

$\|\tilde{\mu}(t)\| \equiv 0, \forall t>T=\frac{2 L}{\bar{\rho} \underline{x} v} \sqrt{\lambda}\|\tilde{\mu}(0)\|$

where it has been supposed without loss of generality that the observer was initialized with $\hat{x}(0)=x(0)$.

This finishes the proof.

Note. Observer (5) captures the dynamic structure of (4), thus leading to an error dynamics amenable for the convergence proof. Of course, other variations of the super-twisting algorithm can be alternatively proposed, but more involved stability analysis and too conservative gains will probably arise.

\section{Simulation results}

This section presents a pair of numerical examples that illustrate the previous analysis and theoretical results. In the next section, experimental data is provided to assess the observer performance in a realistic scenario.
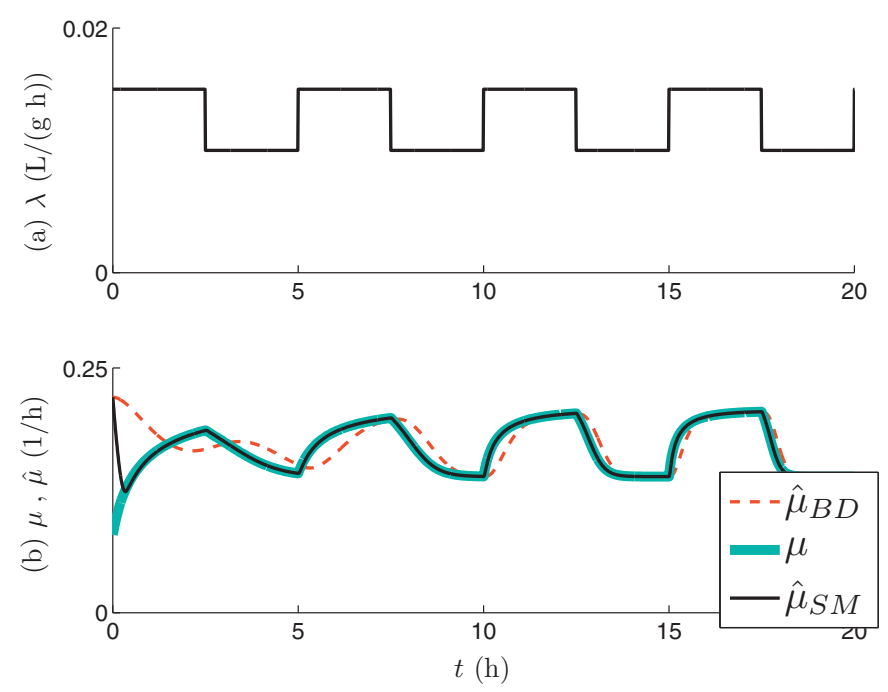

Fig. 2. Open-loop simulation results. (a) Input signal $\lambda(t)$. (b) Real $(\mu)$ and estimated growth rate using adaptive $\left(\hat{\mu}_{B D}\right)$ and sliding $\left(\hat{\mu}_{S M}\right)$ observers.

Let consider the fed-batch process

$\mathcal{P}_{E}:\left\{\begin{array}{l}\dot{x}=(\mu(s)-\lambda(t) x) x \\ \dot{s}=\left(-y_{s} \mu(s)+\lambda(t)\left(s_{i}-s\right)\right) x\end{array}\right.$

with haldane kinetics $\mu(s)=\mu_{m}\left(s /\left(k_{s}+s+s^{2} / k_{i}\right)\right)$ and feeding profile $D(x, t)=\lambda(t) x$. The parameters are $\mu_{m}=0.22, k_{s}=0.14, k_{i}=0.4$, $y_{s}=1.43$ and $s_{i}=20$. Note that (23) can be rewritten as (1) after the change of variable $(x, s) \mapsto(x, \mu(s))$.

For comparative purposes, both the adaptive (2) and the proposed sliding observer (5) have been implemented to estimate $\mu$.

\subsection{Open-loop simulation}

The process input $\lambda(t)$ is a piece-wise constant signal switching every $2.5 \mathrm{~h}$. Observers (2) and (5) are tuned with $\omega=1.5, \zeta=2^{-1 / 2}$, $\bar{\rho}=.4, \alpha=1.1$ and $\beta=1.8$. Their initial conditions are $(\hat{x}(0), \hat{\mu}(0))=$ $\left(x(0), \mu_{m}\right)$. The simulation results are shown in Fig. 2. The top plot depicts the input $\lambda(t)$ whereas the real and estimated specific growth rates are displayed in the bottom plot. The real $\mu(t)$ is shown in solid line (thick trace), the sliding observer estimate is plotted with solid thin trace and the adaptive observer estimate is plotted in dashed line. It is seen that the sliding observer output converges in less than $2 \mathrm{~h}$ and perfectly tracks the evolution of $\mu(t)$ thereafter, whereas the adaptive observer (2) reaches a neighborhood of $\mu(t)$ but does not converge to it. Naturally, since the measurement is not corrupted with noise, the bandwidth of the adaptive observer can be increased to exhibit a faster response. Anyway, the aim of this example is to illustrate qualitatively and comparatively the theoretical convergence features of the proposed sliding observer. Performance under real measurement conditions is evaluated in the next section.

\subsection{Closed-loop simulation}

We present here a closed-loop numerical example to illustrate the potential advantages of the sliding mode observer in closedloop applications. The input signal used in this case is the nonlinear feed-back law:

$\lambda(\mu)=\frac{y_{s} \mu_{r}}{s_{i}-s_{r}}\left(1-k\left(\mu-\mu_{r}\right)\right)$

where $s_{r}$ is the substrate concentration at which $\mu\left(s_{r}\right)=\mu_{r}$. It is shown in [24] that $\lambda(\mu)$ stabilizes the specific growth rate. Moreover, global stability can be achieved even in the presence of kinetic 
Feed-back: $\lambda(\mu)$

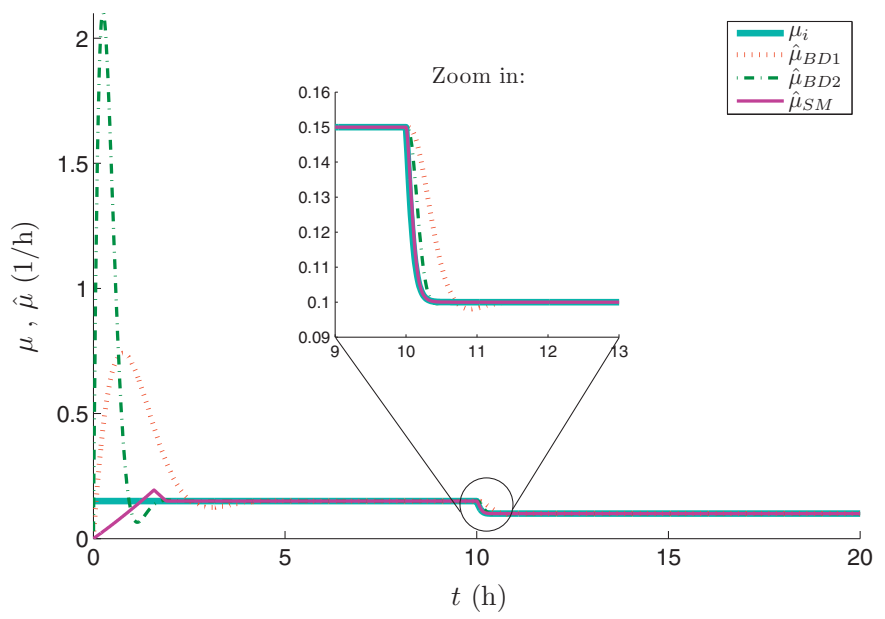

Fig. 3. Closed-loop simulation results when the real $\mu$ is used in the feed-back law (observers are not in loop). $\mu_{i}$ : growth rate using ideal feed-back law (24); $\hat{\mu}_{B D 1}$, $\hat{\mu}_{B D 2}$ : adaptive observer estimates for $\omega=1.5$ and $\omega=4.5$, respectively; $\hat{\mu}_{S M}$ : sliding observer estimate.

multiplicity by properly tuning the feed-back gain $k$. Anyway, the purpose here is not to evaluate the controller performance but the sliding-mode observer one. Then, $\mu(t)$ in (24) is replaced by $\hat{\mu}(t)$. Here, we choose $k=15$.

The simulation run is planned to show the convergence and tracking properties of the observer. With this purpose, the observer is reset at $t=0 \mathrm{~h}$, whereas a set-point step from $\mu_{r}=.15$ to $\mu_{r}=.1$ is produced at $t=10 \mathrm{~h}$.

The tuning parameters of the sliding observer are $\bar{\rho}=.1, \alpha=1.1$ and $\beta=1.8$. In this example, its performance is compared with the performance of observer (2) for two different tunings ( $\omega=1.5$ and $\omega=4.5$ ).

Fig. 3 shows the growth rate and its estimates when the real $\mu-$ not any of its estimates - is used in the feedback law (24). That is, the loop is not closed through an observer. The thick line, labeled with $\mu_{i}$, is the time evolution of the real growth rate. The estimates provided by observer (2) for both tunings and by observer (5) are also plotted. After restarting, the adaptive observer estimates exhibit large overshoots that increase with $\omega$. After the set-point step, the adaptive observer estimate lags the real signal, particularly for low $\omega$. On the other hand, the sliding observer perfectly tracks $\mu(t)$ during the transient that follows the set-point step, whereas initial convergence after restarting is significantly better.

Fig. 4 shows what happens when the observer estimates are used to construct the feeding law. Gaussian noise is added to biomass concentration measurement. The first two plots depict responses obtained with the adaptive observer whereas the remaining ones correspond to the sliding observer. Note that the adaptive observer estimates are out of scale during the first hours after restarting. The top plot shows that the closed-loop response becomes highly oscillatory when the slow adaptive observer is used to close the loop. This is because it adds its slow dynamics to the loop. When the fast adaptive observer is used, oscillations are almost eliminated, but an undesirable initial transient still occurs because of the large observer overshoot. In the third plot, the response obtained with the proposed sliding observer is shown. It is seen that the observer converges rapidly, whereas the tracking response is similar to the fast adaptive observer. The bottom plot illustrates how the sliding function, which continuously switches after convergence, can be used to improve further the initial transient. In this case, the feed-back loop is closed just after the sliding function switches for the first time. Note also that the fast adap-
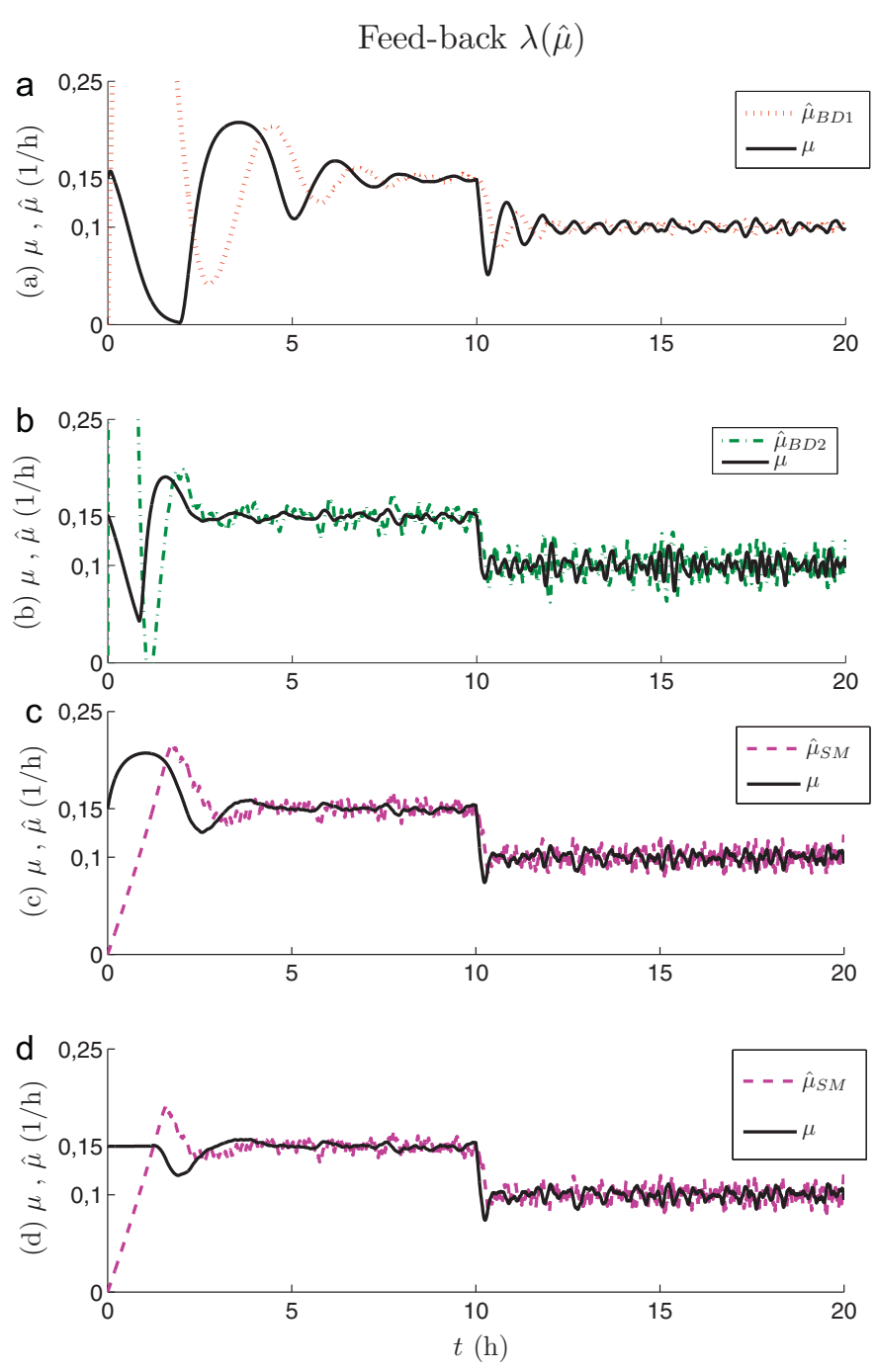

Fig. 4. Closed-loop simulation results when observers are in the loop, noise added to the measured signal. (a) Adaptive observer with $\omega=1.5$, (b) adaptive observer with $\omega=4.5$, (c) sliding observer, and (d) sliding observer in the loop after first switching.

tive observer is noisier than the sliding one. Anyway, since both observers smooth out the measured signal in two different ways, noise performance may differ depending on the noise structure. Performance of these observers in a real scenario is presented in the following section.

\section{Experimental results}

A batch fermentation of the industrial strain Saccharomyces Cerevisiae T73 (wild type) was run. Biomass concentration was measured using the sensor described in [13]. Sampling was carried out each 12 seconds, and a filtered value over a window of 2 min was provided. Growth rate was estimated using the proposed sliding observer, which was tuned as in the previous numerical example, i.e. $\bar{\rho}=0.1, \alpha=1.1$ and $\beta=1$.8. For comparative purposes, a second estimation was obtained using the high-gain observer (2) tuned with $\omega=1.5$. Obviously, the real growth rate is not available to assess the observers performance. We use instead a crude estimate obtained by directly differentiating the measured biomass concentration:

$\mu_{d}(t)=\frac{\dot{x}_{m}(t)}{x_{m}(t)}$ 

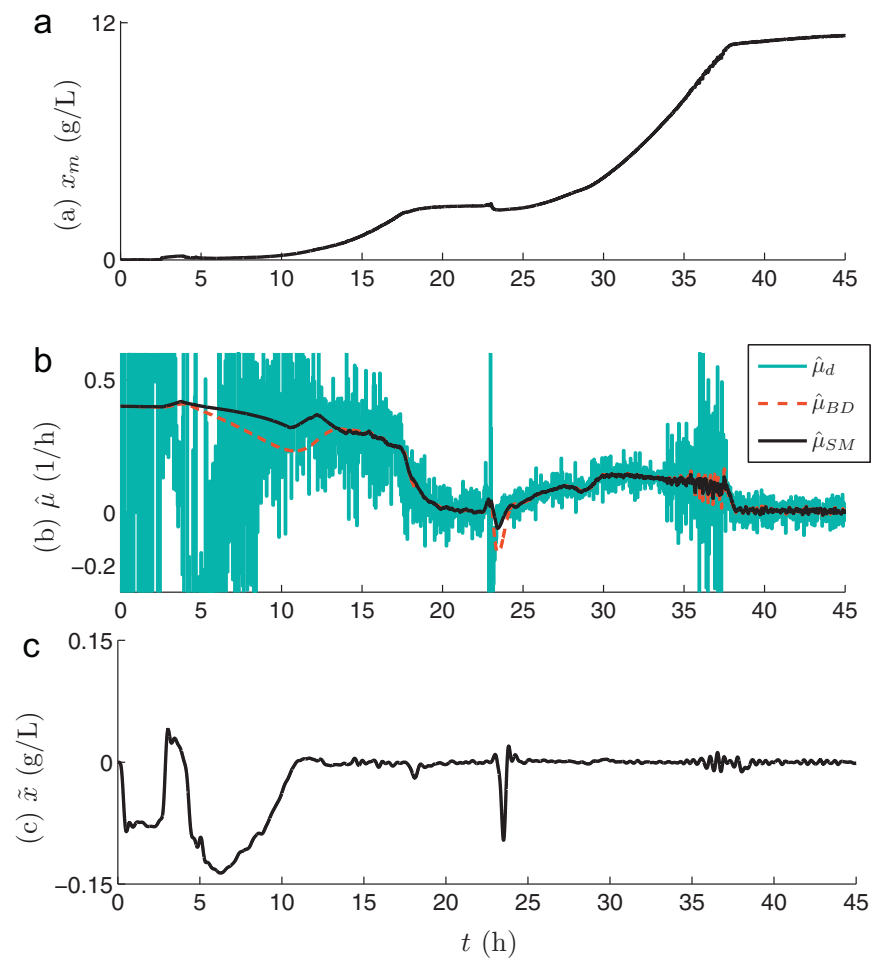

Fig. 5. Experimental results. (a) Measured biomass concentration $\left(x_{m}\right)$.(b) Estimates of the specific growth rate obtained by sensor output differentiation $\left(\hat{\mu}_{d}\right)$ and using $\mathrm{B} \& \mathrm{D}\left(\hat{\mu}_{B D}\right)$ and sliding $\left(\hat{\mu}_{S M}\right)$ observers. (c) Biomass estimation error $(\tilde{x})$ of the sliding observer.

Under the assumption that biomass measurement is accurate and noiseless, $\mu_{d}(t)$ provides the real specific growth rate. In reality, $\mu_{d}(t)$ is highly corrupted with noise. Anyway, in the figures shown below, the real $\mu(t)$ can be guessed behind the noisy $\mu_{d}(t)$.

Fig. 5a plots the evolution of the measured biomass concentration $x_{m}(t)$, whereas Fig. 5b displays the estimates of the specific growth rate obtained from $x_{m}(t)$. The noisiest estimate is $\mu_{d}(t)$. The estimate plotted in dashed line was obtained by the high-gain observer. This estimate coincides with that obtained by smoothing $\mu_{d}(t)$ with a 2 nd-order low-pass filter with time-varying bandwidth $(\omega x(t))$. The estimate is particularly noisy - as measurement is - around $t=35 \mathrm{~h}$. This noise is hardly filtered by the observer because their bandwidths overlap. A lower observer bandwidth would help to reduce noise but at the cost of poorer tracking response. Finally, the signal plotted in solid line is the output of the sliding observer (5) The estimate is smoother than the previous one, particularly around $t=35 \mathrm{~h}$. This is because the observer is less sensitive to fast, and unfeasible, signal gradients. Fig. $5 \mathrm{c}$ displays the biomass estimation error $\tilde{x}(t)$ smoothed out by a low-pass filter, showing that the observer converges in $11 \mathrm{~h}$. During this period, the sliding observer is less sensitive to large measurement errors that are typical of the initial phase of batch processes when biomass concentration is too low.

It is of particular interest to analyze the observer outputs around $t=23 \mathrm{~h}$. As observed in the biomass evolution, the growth almost stops at $t=18 \mathrm{~h}$, most probably due to the depletion of some essential substrate. After that, a pulse of conjugated linoleic acid vaccine was administered at $t=23 \mathrm{~h}$, reactivating the microorganism growth. As seen in Fig. 5b this sudden change in behavior clearly affects both observers. Indeed, from the point of view of the observers, an unpredicted oscillation of the biomass measurement occurred. It is observed that the B\&D observer responds with a large undershoot that vanishes just after $1.5 \mathrm{~h}$. On the contrary, the sliding observer is much less sensitive to this perturbation. In fact, Fig. $5 c$ shows that the observer diverges and then converges rapidly, putting in evidence the occurrence of an abrupt fault. Note that the surface coordinate is an effective residual to indicate bioreactor malfunctions as well as sensor faults or changes in system behavior (both abrupt and gradual). Thus, on one hand, the observer output is less sensitive to the perturbation while on the other its sliding coordinate is very sensitive to it.

\section{Conclusions}

In the article, a second-order sliding mode observer has been developed and analyzed for the estimation of the specific growth rate of microorganisms from measurements of biomass concentration. The resultant observer can be applied to either batch or fed-batch fermentation processes in which the bioreaction exhibits either monotonic or non-monotonic kinetics. Actually, the observer does not use any model of the kinetics of the reaction, just a bound on its time derivative. The proposed observer is based on highgain observers, to which discontinuous correcting terms have been added in order to cancel the estimation error on the measured variable. The structure of the discontinuous output error injection is modified with respect to previous developments, thus providing a smooth estimate without the need of filtering. In contrast with continuous observers, perfect tracking after finite convergence time can be achieved in the absence of noise. Although convergence to a small ball can only be guaranteed in the presence of noise, this theoretical property has important implications in control. In fact the separation principle applies, so that observer and controller in the loop can be designed independently. Simulation and experimental results confirm the distinctive convergence properties of the observer, as well as its potential use in fault detection.

Further research is oriented to the estimation of multiple rates. The main problem is that an extra unknown function must be incorporated to the algorithm in order to avoid too conservative bounds. Stability proof of the generalized algorithm is the key issue. The semi-definite programming approach used in this paper provides powerful tools for this purpose.

\section{Acknowledgements}

This work was supported by the National University of La Plata (Project 11-I127), ANPCyT (PICT2007-00535) and CONICET (PIP112-200801-01052) of Argentina; the Technical University of Valencia (PAID-02-09 program and FPI-2009/21 grant), the CICYT (DPI2005-01180) and AECID (A/024186/09) of Spain; and by FEDER funds of the European Union.

\section{References}

[1] D. Dochain, State and parameter estimation in chemical and biochemical processes: a tutorial, Journal of Process Control 13 (8) (2003) 801-818.

[2] Ch. Venkateswarlu, Advances in monitoring and state estimation of bioreactors, Journal of Scientific \& Industrial Research 63 (2004) 491-498.

[3] S. Aborhey, D. Williamson, State and parameter estimation of microbial growth process, Automatica 14 (1978) 493-498.

[4] G. Bastin, D. Dochain, On-line estimation of microbial specific growth rates, Automatica 22 (1986) 705-709.

[5] J.P. Gauthier, H. Hammouri, S. Othman, A simple observer for nonlinear systems: applications to bioreactors, IEEE Transactions on Automatic Control 37 (6) (1992) 875-880.

[6] M. Farza, K. Busawon, H. Hammouri, Simple nonlinear observers for on-line estimation of kinetic rates in bioreactors, Automatica 34 (3) (1998) 301-318.

[7] V. Lemesle, J.L. Gouzé, Hybrid bounded error observers for uncertain bioreactor models, Bioprocess and Biosystem Engineering 27 (2005) 311-318.

[8] Ph. Bogaerts, X. Hulhoven, A. Vande Wouwer, Hybrid extended luenbergerasymptotic observer for bioprocess state estimation, Chemical Engineering Science 21 (2006) 7151-7160.

[9] A. Nagy-Kiss, B. Marx, G. Mourot, G. Schutz, J. Ragot, State estimation of twotime scale multiple models with unmeasurable premise variables. Application to biological reactors, in: Proceedings IEEE Conference on Decision and Control, 2010, pp. 5689-5694. 
[10] J. González, G. Fernández, R. Aguilar, M. Barron, J. Álvarez-Ramírez, Sliding mode observer-based control for a class of bioreactors, Chemical Engineering Journal 83 (2001) 25-32.

[11] J. Picó, H. De Battista, F. Garelli, Smooth sliding-mode observers for specific growth rate and substrate from biomass measurement, Journal of Process Control 19 (8) (2009) 1314-1323, Special Section on Hybrid Systems: Modeling, Simulation and Optimization.

[12] N. Zamboni, S.M. Fendt, M. Rühl, U. Sauer, 13c-based metabolic flux analysis, Nature Protocols 4 (2009) 878-892.

[13] J. Navarro, J. Picó, J. Bruno, E. Picó-Marco, S. Vallés, On-line method and equipment for detecting, determining the evolution and quantifying a microbial biomass and other substances that absorb light along the spectrum during the development of biotechnological processes (patents ep20020751179, and us patent 6975403). Technical report, 2001.

[14] K. Kiviharju, K. Salonen, U. Moilanen, T. Eerikainen, Biomass measurement online: the performance of in situ measurements and software sensors, Journal of Industrial Microbiology and Biotechnology 35 (7) (July 2008) 657-665.

[15] A. Levant, Robust exact differentiation via sliding mode technique, Automatica 34 (3) (1998) 379-384.

[16] G. Bastin, D. Dochain, On-line Estimation and Adaptive Control of Bioreactors, Elsevier, Amsterdam, The Netherlands, 1990.
[17] I. Dunn, E. Heinzle, J. Ingham, J. Přenosil, Biological Reaction Engineering Dynamic Modelling Fundamentals with Simulation Examples, Wiley-VCH Verlag, 2003.

[18] J. Dávila, L. Fridman, A. Levant, Second-order sliding-mode observer for mechanical systems, IEEE Transactions on Automatic Control 50 (11) (2005) 1785-1789.

[19] J. Moreno, M. Osorio, A Lyapunov approach to second-order sliding mode controllers and observers, in: Proceedings of the 47th IEEE Conference on Decision and Control, 2008.

[20] L. Fridman, A. Levant, Sliding Mode Control in Engineering, Chapter Higherorder Sliding Modes, Marcel Dekker, Inc., 2002, pp. 53-101.

[21] A. Levant, Higher-order sliding modes, differentiation and output-feedback control, International Journal of Control 76 (9/10) (2003) 924-941.

[22] S. Boyd, L. El Ghaoui, E. Feron, V. Balakrishnan, Linear Matrix Inequalities in System and Control Theory, Society for Industrial and Applied Mathematics, 1994.

[23] J. Löfberg, Yalmip: a toolbox for modeling and optimization in MATLAB, in: Proceedings of the CACSD Conference, Taipei, Taiwan, 2004.

[24] H. De Battista, J. Picó, E. Picó-Marco, Globally stabilizing control of fed-batch processes with haldane kinetics using growth rate estimation feedback, Journal of Process Control 16 (2006) 865-875. 